\title{
Influence of Hydrolysis Conditions on Characteristics of Nanocrystalline Cellulose Extracted from Ramie Fibers by Hydrochloric Acid Hydrolysis
}

Kusmono Kusmono ( $\nabla$ kusmono@ugm.ac.id)

Universitas Gadjah Mada https://orcid.org/0000-0003-1465-3013

Dimas Abdillah Akbar

Universitas Gadjah Mada

\section{Research}

Keywords: ramie fibers, nanocrystalline cellulose, hydrochloric acid hydrolysis

Posted Date: June 11th, 2020

DOI: https://doi.org/10.21203/rs.3.rs-31322/v1

License: (c) (1) This work is licensed under a Creative Commons Attribution 4.0 International License. Read Full License 


\section{Abstract}

Nanocrystalline celluloses (NCCs) were successfully extracted from ramie fibers using chemical pretreatments followed by hydrochloric acid hydrolysis. The effects of acid concentration and hydrolysis time on the characteristics of NCCs were investigated in this study. Results showed that the optimal hydrolysis conditions were found to be $6 \mathrm{M}$ hydrochloric acid concentration at $45^{\circ} \mathrm{C}$ for $70 \mathrm{~min}$. The obtained NCC had a rod like-shape with an average of $8.07 \mathrm{~nm}$ in diameter, $158.51 \mathrm{~nm}$ in length, $22.37 \mathrm{in}$ aspect ratio, $89.61 \%$ in the crystallinity index, and $5.81 \mathrm{~nm}$ in crystallite size. The higher crystallinity and thermal stability were exhibited by NCCs compared to both raw fibers and chemically purified cellulose. The hydrolysis time had a significant effect on crystallinity and thermal stability. The crystallinity index and thermal stability of NCCs were obtained to decrease with increasing hydrolysis time.

\section{Introduction}

In recent years, rapid developments and many opportunities for nanotechnology attracted special attention from researchers and industrialists, especially in nanocrystalline cellulose (NCC) [Khalil et al. 2012; Peng et al. 2011; Mishra et al. 2019]. That is due to its superior properties such as high specific strength, high specific modulus, high flexibility, good dynamic mechanical and unique optical properties, low coefficient of thermal expansion, large specific surface area, nanoscale dimension, high aspect ratio, low cost, biodegradable, and biocompatibility [Chen et al. 2011; Moriana et al. 2016; Wang et al. 2019]. Those properties bring NCC to be nano reinforcement materials for the nanocomposites for many suitable applications such as packaging, construction, and biomedicine, reinforcement materials in flexible light-emitting diode panels, oxygen barrier layers, pharmaceutical, and food industry [Chen et al. 2011; Moriana et al. 2016; Brinchi et al. 2013].

NCC is a nano-sized material with the structure of rigid rod-like shaped whiskers with a diameter in the range of 1-100 nm and a length in the range of 10-100 nm [Fan et al. 2012; Zainuddin et al. 2017]. NCC is extracted from cellulose that contains amorphous and crystalline regions. Moreover, NCC has a good ordered crystalline structure due to the amorphous zone of cellulose is dissolved during processing [Pirani et al. 2013; Naduparambath et al. 2018]. NCC can be prepared from cellulosic materials such as bacteria, algae, animal (tunicate), plants, and some waste material [Jasmani and Adnan 2017; llyas et al. 2018; Lei et al. 2018]. Plants are the most common materials, which is used for NCC extraction due to their high cellulose content [Brinchi et al. 2013; Oun and Rhim 2016].

The common plants used for NCC extraction are wood fibers, cotton, sugar cane, wheat straw, hemp, sisal, jute, coir, and flax fibers [Chen et al. 2011; Bledzki and Gassan 1999]. The obtained NCC from the natural biopolymer materials is renewable, environmentally friendly, bio-degradable, non-toxic, and extremely abundant in the earth [Oun and Rhim 2016; Sun et al. 2018]. Ramie fiber is one of the natural biopolymers that has a higher tensile strength compared to sisal, jute, coir, flax, and cotton. Moreover, ramie fibers also have higher cellulose content and crystallinity index compared to jute, hemp, flax, coir, and sisal [Bledzki and Gassan 1999]. 
Extracting NCC from lignocellulose materials can be done through chemical pretreatment (cellulose purifying) to remove amorphous regions (wax, lignin, pectin, and hemicellulose) followed by acid hydrolysis. Some commonly used mineral acids include phosphoric acid, sulfuric acid, hydrochloric acid, and their mixtures [Jasmani and Adnan 2017; Liu et al. 2014]. Cellulose hydrolysis aims to eliminate amorphous cellulose areas to obtain cellulose crystalline regions [Oun and Rhim 2016]. The most popular method is acid hydrolysis by sulfuric acid [Rosa et al. 2010] where this method causes the extracted NCC to have a unique surface property of $\mathrm{SO}_{3}{ }^{-}$that makes $\mathrm{NCC}$ have a good dispersion in water solvent [Yu et al. 2013]. However, the phenomenon will decrease the thermal stability of NCC [Corrêa et al. 2010]. On the other hand, the extraction of NCC through hydrochloric acid hydrolysis can produce a minimal surface charge in extracted NCCs [Oun and Rhim 2015], and the thermal stability of NCC is higher than NCC extracted by sulfuric acid [Corrêa et al. 2010]. Therefore, hydrochloric acid was chosen to obtain NCC with good thermal stability in this work. To date, a study on isolation and characterization of NCC from ramie fibers by hydrochloric acid hydrolysis has not yet been reported.

The characteristics of extracted NCC depends on the raw material, conditions of pretreatment, extraction method, and extraction parameter [Chen et al. 2011; Oun and Rhim 2015; Habibi et al. 2010]. There are several hydrolysis parameters (type and acid concentration, hydrolysis time, hydrolysis temperature, and acid-to-fiber ratio) that affect the characteristics of the obtained NCC [Ditzel et al. 2017; Liu et al. 2017]. To obtain NCC with good characteristics, we need to find the best hydrolysis parameters. The influences of acid concentration and reaction duration on the characteristics (structural, crystallinity index, crystallite size, morphological, and thermal stability) of nanocrystalline cellulose isolated from ramie fibers by hydrochloric acid hydrolysis were investigated in this work.

\section{Experimental}

\subsection{Materials}

Dried ramie fibers (Boehmeria nivea) from ramie's stem plant were purchased from Balai Penelitian Tanaman Pemanis dan Serat (BALITTAS) Malang, East Java, Indonesia. Hydrochloric acid (37.8\%) was purchased from Mallinckrodt, Ireland while toluene (99.9\%), acetic acid (100\%), sodium chlorite (25\%), sodium hydroxide (99\%) were obtained from Merck, USA. Ethanol (99.5\%) was supplied from SigmaAldrich, USA.

\subsection{Isolation of nanocrystalline cellulose}

Before isolation of nanocrystalline cellulose, the ramie fibers were chemically pretreated to obtain chemically purified cellulose (CPC) through chemical pretreatments (de-waxing, bleaching, and alkali treatments) as described in our previous study [Listyanda et al. 2020]. The obtained CPC was then isolated for producing NCC using hydrochloric acid hydrolysis as described in the literatures [Yu et al. 2013; Kumar et al. 2014]. Briefly, a certain amount of distilled water was poured in a beaker glass and dropped by hydrochloric acid (37.8\%) that was placed on the ice water bath under the continuous stirring 
on the magnetic stirrer until the final $\mathrm{HCl}$ concentration was $6 \mathrm{M}$ at $20 \mathrm{ml}$ acid solution. At the end of acid addition, the ice bath was removed, and the acid solution was preheated until $45^{\circ} \mathrm{C}$. After that, $1 \mathrm{~g}$ of CPC was added and kept for $70 \mathrm{~min}$. In this work, the effects of two hydrolysis conditions (acid concentration and reaction time) on the characteristics of NCCs were investigated. A series of NCC suspensions were prepared using various hydrochloric acid concentrations $(6,8$, and $12 \mathrm{M})$ at $45^{\circ} \mathrm{C}$ for 70 min and they were then denoted as NCC-6, NCC-8, and NCC-12, respectively. In addition, NCCs were also prepared with $6 \mathrm{M} \mathrm{HCl}$ at $45^{\circ} \mathrm{C}$ using three hydrolysis times which are 70,125 , and $180 \mathrm{~min}$, denoted as NCC-70, NCC125 , and NCC-180, respectively.

After hydrolysis processes were completed, the suspensions were quenched into the chilled distilled water (temperature $\pm 5^{\circ} \mathrm{C}$ ) with a ratio of suspensions to chilled water 1:20 (v/v) to end the hydrolysis process. The suspensions were then centrifuged at $4,000 \mathrm{rpm}$ for $15 \mathrm{~min}$ to release the acidic solution. The supernatant was then removed and replaced by distilled water followed by centrifugation at 4,000 rpm for 15 min to optimize the removal of the acidic solution. After centrifugation, the NCC sediment was collected and then re-suspended by distilled water and dialyzed against distilled water until neutral $(\mathrm{pH}$ 7). After that, the NCC suspensions were ultrasonicated for 2 min with a $50 \%$ amplitude to produce the homogeneous NCC suspensions. Furthermore, some parts of NCC suspensions were collected and others were freeze-dried for characterizations.

\subsection{Characterizations}

\subsubsection{Chemical composition}

The chemical composition analysis of the fibers was performed to determine the content of the three main components of the fibers, namely: a-cellulose, hemicellulose, and lignin. This was carried out on the raw fibers and CPC. The chemical composition analysis was performed following SNI 0492: 2008 standard.

\subsubsection{Fourier Transform Infra-Red (FT-IR)}

FT-IR spectra were recorded using the IRPrestige21 machine in the range of 4000 to $500 \mathrm{~cm}-1$ were used to analyze the functional group of samples. Two milligrams of samples were mixed with $200 \mathrm{mg} \mathrm{KBr}$ then mixed using a vibrating mill for 30 seconds to make a homogeneous mixture, the mixture was then compacted under vacuum condition to form to pellets and then put into the FTIR machine to obtain its spectra.

\subsubsection{X-Ray Diffraction (XRD)}

XRD was used to calculate the crystallinity index and the crystallite size of the samples. The XRD patterns were analyzed using Empyrean (Malvern PANanalytical) in range of $2 \theta=10$ to $45^{\circ}$ using Nifiltered CuKa ray radiation $(\lambda=0.154 \AA$ ) operated at $40 \mathrm{kV}$ and $30 \mathrm{~mA}$ in room temperature. Furthermore, those patterns were used to calculate the crystallinity index and crystallite size of the samples. The 
crystallinity index of the samples was determined by using the Segal method [Segal et al. 1959] that can be seen in Eq. 1:

$$
C I=\frac{I_{200}-I_{a m}}{I_{200}} \times 100 \%
$$

where $I_{200}$ is the peak intensity of the diffraction at (200) peak for cellulose $I$ at around $2 \theta=22^{\circ}$ and $I_{a m}$ is the intensity related to the amorphous scattering at around $2 \theta=18^{\circ}$. The crystallite size of the samples was determined by using the Scherrer equation [31] following Eq. 2 as follows:

$$
t=\frac{K \lambda}{\beta_{1 / 2} \cos \theta}
$$

where $t$ is the crystallite size, $K$ is the Scherrer constant $(0,91), \lambda$ is the $X$-ray wavelength $(\lambda=1.54060 \AA)$, $\beta_{1 / 2}$ is the full width at the half maximum (FWHM) of the XRD peak in radian and $\theta$ is the Bragg's angle.

\subsubsection{Scanning Electron Microscopy (SEM)}

SEM was used to observe the effect of chemical pretreatment on the surface morphology of the fibers. This observation was carried out on the raw fibers and CPC using ZEISS type EVO MA 10 - SEM with an accelerating voltage of $5 \mathrm{kV}$. Before observing, the samples were coated with palladium by a sputtering method to create a conductive thin layer on the surface of samples.

\subsubsection{Transmission Electron Microscopy (TEM)}

The morphology and dimension of NCC were observed using TEM (JEOL type JEM-1400 Electron Microscope) at an accelerating voltage of $120-200 \mathrm{kV}$ in several different magnifications. Firstly, the NCC suspension was homogenized using an ultrasonic homogenizer for 60 seconds. A drop of the NCC solution was then deposited on a carbon-coated copper grid which was dried and observed for imaging. The diameter and length of NCCs were measured by an image processing analysis program, Image $\mathrm{J}$, using the TEM images.

\subsubsection{Thermal Analysis}

The thermal stability was characterized by using thermogravimetry analysis (TGA) (LINSEIS machine Type TA PT 1600). The experiments were performed at a heating rate of $10^{\circ} \mathrm{C} / \mathrm{min}$ from temperatures of 30 to $600{ }^{\circ} \mathrm{C}$ under the nitrogen atmosphere at a flow rate of 4 liters/hour. Besides, the derivative thermogravimetry (DTG) was obtained from TGA data by calculating using a central finite difference method that can be seen in Eq. 3: 


$$
D T G=\frac{w_{t+\Delta_{t}}-w_{t-\Delta_{t}}}{2 \Delta_{t}}
$$

where $\mathrm{w}_{t+\Delta t}$ is the weight of the sample at time $t+\Delta t$, wt- $\Delta t$ is the weight of sample at time $t-\Delta t$, and $\Delta t$ is the time interval for reading residual sample weight [Oun and Rhim 2015].

\section{Results And Discussion \\ 3.1. Chemical composition}

From the chemical composition analysis, it was found that the raw fibers consist of $72.68 \%$ a-cellulose, $13.70 \%$ hemicellulose, $0.38 \%$ lignin, and $13.24 \%$ others (water, wax, and pectin). This suggests that ramie fiber has high cellulose content and has the potential to be used as a renewable source of cellulose. Therefore, this was the reason for choosing of ramie fibers as a source of cellulose for the preparation of nanocrystalline cellulose. Furthermore, the CPC compose of $92.30 \%$ a-cellulose, $7.18 \%$ hemicellulose, $0 \%$ lignin, and $0.52 \%$ others. This means that the chemical purification significantly raised the a-cellulose and reduced the contents of amorphous regions such as hemicellulose, lignin, wax, and pectin. It is wellknown that the highest polymerization degree and the most stable among all classes of cellulose is acellulose [Dungani et al. 2017]. The increase in a-cellulose content was associated with the removal of amorphous components in the ramie fibers during the process of chemical purification of cellulose [llyas et al. 2018]. The increase of cellulose and the removal of some amorphous part of ramie fibers after cellulose purification resulted in a higher crystallinity index of cellulose that can enhance the strength, stiffness, and the thermal stability of the fiber [Alemdar and Sain 2008].

\subsection{Fourier Transform Infra-Red (FT-IR) Spectroscopy}

The FT-IR spectra of raw fibers, CPC, and NCC hydrolyzed at an acid concentration of $6 \mathrm{M}$ at $45^{\circ} \mathrm{C}$ for 70 min are shown in Fig. 1. No significant change in peaks was exhibited between the spectra of all samples. The strong broad peak at around $3400 \mathrm{~cm}^{-1}$ was related to the stretching vibration of $\mathrm{O}-\mathrm{H}$ groups [Tang et al. 2014]. The peak at $2368 \mathrm{~cm} \otimes^{1}$ was attributed to the stretching vibration of the C-O group. The peak at $1643 \mathrm{~cm}^{-1}$ was mainly attributed to the aromatic ring present in lignin and the vibration binding of water molecules absorbed into fiber structure [Kumar et al. 2014]. The intensity of this peak was decreased in the NCC spectra indicating the removal of the portion of lignin during hydrolysis. For raw fiber, the peak observed at $1740 \mathrm{~cm}^{-1}$ was related to the $\mathrm{C}=0$ bonds of the carboxylic group of hemicelluloses and pectin whereas the small peak at $1506 \mathrm{~cm}^{-1}$ was associated with $\mathrm{C}=\mathrm{C}$ group stretching of the aromatic ring of the lignin [Rosa et al. 2010]. These peaks disappeared in the spectra of CPC and NCC indicating parts of hemicellulose and lignin were removed from ramie fibers during chemical purification and acid hydrolysis [Sheltami et al. 2012]. The peaks at 1371 and $1427 \mathrm{~cm}^{-}$

${ }^{1}$ corresponded to the $\mathrm{C}-\mathrm{H}$ bonds symmetrical angular deformation and $\mathrm{C}-\mathrm{H}$ bonds asymmetrical angular 
deformation, respectively [Kian et al. 2018]. The peaks at $2903 \mathrm{~cm}^{-1}$ (C-H symmetrical stretching), 1160 $\mathrm{cm} \rrbracket^{1}$ (C-O-C glycoside bonds asymmetrical stretching), $1111 \mathrm{~cm}^{-1}$ (C-OH stretching), $897 \mathrm{~cm}^{-1}$ ( $\beta-$ glycosidic bonds bending) presented to the cellulose I characteristics was more obvious for NCC. This reveals the NCC had the highest cellulose content among all samples. For NCC, the peak at $1058 \mathrm{~cm} \mathbb{1}^{1}$ (CO-C pyranose ring vibration) displayed sharper compared to others indicating the higher cellulose content for NCC [Ngwabebhoh et al. 2018]. It can be concluded that the chemical purification and hydrochloric acid hydrolysis removed some parts of amorphous components such as lignin, hemicellulose, and wax of fibers. FTIR results showed the cellulose $I \beta$ structure and the hydrochloric acid hydrolysis resulted in higher cellulose content than both raw fiber and CPC.

\subsection{X-Ray Diffraction (XRD)}

Figure 2 displays the X-ray diffraction patterns of the raw fibers, CPC, and NCCs obtained under different hydrolysis conditions. Figure $2 \mathrm{a}$ demonstrates the effect of acid concentration on the diffraction patterns of NCCs hydrolyzed at $45^{\circ} \mathrm{C}$ for $70 \mathrm{~min}$. The influence of reaction time on the diffraction patterns of NCCs hydrolyzed with the $6 \mathrm{M}$ hydrochloric acid solution at $45^{\circ} \mathrm{C}$ is presented in Fig. $2 \mathrm{~b}$. All samples showed a similar four characteristic peaks detected at $2 \theta=15,16,22$, and $34^{\circ}$ corresponding to cellulose I with assigned planes of (1 10), (110), (200), and (004), respectively. From Fig. 2, it can be concluded that the acid concentration and hydrolysis time did not influence the crystal structure of the raw fiber as indicated with no different peaks for all samples. These results are consistent with the FT-IR spectra as shown in Fig. 1. Similar findings were also demonstrated by previous researchers [Ngwabebhoh et al. 2018] where the crystal structure of cellulose was not influenced by the acid concentration, temperature, and reaction times during hydrolysis.

From Fig. 2, the crystallinity index of the samples is then calculated using the Segal equation [Segal et al. 1959] and the results are summarized in Table 1. The crystallinity index values of raw fiber and CPC are 79.75 and $86.68 \%$, respectively. The slight increase was attributed to the removal of hemicellulose and lignin during the chemical purification process of cellulose [Sheltami et al. 2012]. The effect of hydrochloric acid concentration on the crystallinity index of NCCs as indicated by NCC-6, NCC-8, and NCC12 is also presented in Table 1. It can be seen that NCCs had slightly higher values of the crystallinity index compared to both raw fiber and CPC. The further removal of amorphous regions during acid hydrolysis and subsequent hydrolytic cleavage of glycosidic bonds and releasing of individual crystallites might be responsible for the slight increase of the crystallinity [Lu and Hsieh 2012; Owolabi et al. 2016]. From Table 1, it can also be found that there is no significant difference in the crystallinity under different acid concentrations where the highest value in the crystallinity (89.61\%) was exhibited at $6 \mathrm{M}$ chloric acid concentration. This suggests that the acid concentration did not significantly influence the crystallinity index of NCC during acid hydrolysis. The crystallinity index values of NCCs hydrolyzed with the reaction times of 70,125 , and 180 min is $89.61,84.07$, and 82.60 , respectively. The crystallinity was decreased with increasing reaction time. At longer reaction time, the hydrogen ions not only removed the amorphous areas but also the cellulose crystalline areas to encourage further hydrolytic cleavage of the glycosidic 
bonds leading to the decreased crystallinity index [Al-Dulaimi and Wanrosli 2017]. Similar observations were also reported by previous researchers [Al-Dulaimi and Wanrosli 2017; Lei et al. 2018] where the crystallinity was reduced with increasing reaction time and more than 80 min led to significant damage to the crystal. In this work, it can be concluded that the highest crystallinity value (89.6\%) was obtained for NCC prepared with the hydrolysis conditions of $6 \mathrm{M}$ hydrochloric acid concentration at $45^{\circ} \mathrm{C}$ for $70 \mathrm{~min}$ (NCC-6). This crystallinity index of NCC-6 (89.6\%) was higher than pineapple leaves 54\% [Cheriana et al. 2010], pueraria root $60 \%$ [Wang et al. 2019], soy hulls 73.5\% [Neto et al. 2013], sisal 78\% [Brito et al. 2012], sugar palm 85.9\% [llyas et al. 2018], curaua 87\% [Brito et al. 2012], bamboo 87\% [Brito et al. 2012], eucalyptus $89 \%$ [Brito et al. 2012] but slight lower than cotton linter $90 \%$ [Morais et al. 2013] and corn husk $92 \%$ [Mendes et al. 2015]. The crystallinity index is strongly influenced by several factors such as types of fibers, purification time of fibers, and hydrolysis conditions (type and concentration of acids, temperature, and reaction time) [llyas et al. 2018]. The number of crystallinity degree region and the mechanical properties of cellulose were are interrelated where the mechanical properties (stiffness and tensile strength) of fibers will increase as the number of crystallinity region increases [llyas et al. 2018]. Therefore, the mechanical properties of nanocomposite materials can be improved by the addition of nanocrystalline cellulose with higher crystallinity index [Bhatnagar et al. 2005]. The obtained NCC has great potential as reinforcement material in nanocomposites due to high its crystallinity index.

Table 1

Crystallinity index and crystallite size of raw fiber, CPC and NCCs under different hydrolysis conditions

\begin{tabular}{|lll|}
\hline Sample & Crystallinity index (Cl) & $\begin{array}{l}\text { Crystallite size } \\
(\mathrm{nm})\end{array}$ \\
\hline Raw fiber & 79.75 & 3.96 \\
\hline CPC & 86.68 & 4.75 \\
\hline NCC-6 & 89.61 & 5.81 \\
\hline NCC-8 & 89.23 & 5.51 \\
\hline NCC-12 & 87.53 & 5.08 \\
NCC-70 & 89.61 & 5.81 \\
NCC-125 & 84.07 & 3.36 \\
NCC-180 & 82.60 & 3.34 \\
\hline
\end{tabular}

From the XRD patterns, the crystallite size of cellulose, and NCCs was determined by using the Scherrer equation and the values are demonstrated in Table 1. The crystallite size of raw fiber and CPC was 3.96 and $4.75 \mathrm{~nm}$, respectively. The higher crystallite size of CPC compared to that of raw fiber was ascribed to the removal of amorphous lignin and hemicellulose during purification as shown in the FT-IR spectra [Sheltami et al. 2012]. Compared with the raw fiber and CPC, the crystallite size of all NCCs was higher. This might be related to the narrowed distribution of the crystallite size [Lu and Hsieh 2012]. It can also 
be observed from Table 3 that the crystallite size slightly decreased with increasing the acid concentration. A similar trend could also be observed when the reaction time increased where the crystallite size was also reduced. A higher reduction in the crystallite size was exhibited by the increased reaction time compared to acid concentration. This indicates the reaction time had a higher effect than the acid concentration in this study. The dissolution of both the amorphous areas and part of crystalline domains during hydrolysis might be responsible for the reduced crystallite size with increasing the acid concentration and reaction time [Samir et al. 2004]. Similar findings were also demonstrated by previous researchers [Lei et al. 2019] where the crystallite size of NCCs decreased by increasing the sulfuric acid concentration.

Table 3

Onset temperature $\left(T_{0}\right)$, degradation temperature on maximum weight loss rate $\left(\mathrm{T}_{\max }\right)$ and char yield $\left(\mathrm{W}_{\text {residue }}\right)$ for raw fiber, CPC and NCCs under different hydrolysis conditions obtained from TGA and DTG curves

\begin{tabular}{|llll|}
\hline Sample & TGA & & DTG \\
\cline { 2 - 4 } & TQ $\left({ }^{\circ} \mathrm{C}\right)$ & $\mathrm{W}_{\text {residue }}(\%)$ & $\mathrm{T}_{\max }\left({ }^{\circ} \mathrm{C}\right)$ \\
\hline Raw fiber & 158 & 4 & 346 \\
\hline CPC & 242 & 1 & 337 \\
\hline NCC-6 & 254 & 10 & 332 \\
\hline NCC-8 & 252 & 10 & 328 \\
\hline NCC-12 & 253 & 11 & 330 \\
\hline NCC-70 & 254 & 10 & 332 \\
\hline NCC-125 & 233 & 19 & 354 \\
\hline NCC-180 & 235 & 11 & 357 \\
\hline
\end{tabular}

Figure 3 displays the SEM micrographs of raw fibers and chemically purified cellulose (CPC). The chemical treatments were done through de-waxing, bleaching, and alkali treatments. The raw fibers were consists of the individual fibers linked together as bundles by cement materials (Fig. 3a). The raw fiber had a rough surface layer with the presence of high amorphous components such as hemicellulose, lignin, wax, pectin, and oil [Fardioui et al. 2016]. Furthermore, de-waxing, bleaching, and alkali treatments allowed to remove the amorphous components such as hemicellulose and lignin present in the untreated ramie fibers, resulting in defibrillation of raw fibers to the cellulose fibers (Fig. 3b). Therefore, the average diameter of fibers was decreased from $60 \mu \mathrm{m}$ for the raw fibers to $45 \mu \mathrm{m}$ for CPC. Similar findings were also demonstrated by previous researchers where the bleaching and alkali treatments reduced the diameter of raw fibers [Rosli et al. 2013; Fardioui et al. 2016]. Visually, the raw fibers had a yellowish color whereas CPC was a pure white. This confirms the success of cellulose purification through chemical 
treatments. Furthermore, the morphology of obtained NCCs was observed by using the TEM examination and discussed later.

\subsection{Transmission Electron Microscopy (TEM)}

Figure 4 displays the TEM images of NCC-6, NCC-12, and NCC-180, respectively. NCC-6 was NCC prepared under hydrolysis with $6 \mathrm{M} \mathrm{HCl}$ at $45^{\circ} \mathrm{C}$ for 70 min whereas NCC-12 was produced from the hydrolysis with $12 \mathrm{M} \mathrm{HCl}$ at $45^{\circ} \mathrm{C}$ for 70 min. NCC-180 presented NCC hydrolyzed with $6 \mathrm{M} \mathrm{HCl} 45^{\circ} \mathrm{C}$ for $180 \mathrm{~min}$. A rod or needle-like structure in a nanoscale dimension of NCC could be observed in Fig. 4. All NCCs exhibited almost similar morphology where NCCs were not fully dispersed individually but agglomeration tendency. This is due to the lack of an electrostatic repulsion charge between crystal particles because chlorite ions are easily removed during the dialysis process [Lima and Borsali 2004; Roman and Winter 2004; Wang et al. 2007]. In addition, NCC particles hydrolyzed by HCl have a strong hydrogen bonding at the surface of NCC particles resulting in agglomeration [Cheng et al. 2017]. Similar observations were also reported by previous researchers [Corrêa et al. 2010] where NCCs particles hydrolyzed with hydrochloric acid was in agglomeration. From TEM observation, it could be concluded that the acid concentration and reaction time did not affect significantly the morphology of NCCs as indicated no significant difference in the morphology of NCCs.

The diameter and length distribution of NCCs particles measured from TEM images are shown in Fig. 5. The dimensions of NCCs were analyzed by measuring particle length (L), diameter (D), aspect ratio (L/D), and the results are summarized in Table 2. The diameter and length of NCCs were calculated by an image processing analysis program, Image $\mathrm{J}$ using TEM images. Figures $5 a-b$ display the distribution of diameter and length of NCC prepared at $45^{\circ} \mathrm{C}$ for 70 min with hydrochloric acid $6 \mathrm{M}$ (NCC-6), respectively. The diameter and length distribution of NCC- 6 was about $4-13 \mathrm{~nm}$ and $110-210 \mathrm{~nm}$, respectively. The average distribution of NCC- 6 was $8.07 \pm 1.73 \mathrm{~nm}$ in diameter and $160.22 \pm 21.59 \mathrm{~nm}$ in length. Then, the diameter and length distribution of NCC particles produced under $12 \mathrm{M}$ hydrochloric acid at $45^{\circ} \mathrm{C}$ for $70 \mathrm{~min}$ (NCC-12) was about 3-10 nm and 110-170 nm (Figs. 5c-d), respectively. The average distribution of NCC-12 was $6.77 \pm 1.30$ in diameter and $144.49 \pm 12.64$ in length. Figures $5 \mathrm{e}-\mathrm{f}$ display the diameter and length distribution of NCC particles prepared under $6 \mathrm{M}$ hydrochloric acid at $45^{\circ} \mathrm{C}$ for $180 \mathrm{~min}$ (NCC-180) where the diameter and length distribution were around $3-6 \mathrm{~nm}$ and $60-200 \mathrm{~nm}$. The average distribution of NCC-180 was $4.55 \pm 0.89$ in diameter and $109.23 \pm 28.97$ in length. From Fig. 5 , it can be seen that NCC- 6 and NCC-12 exhibited almost similar in the distribution of diameter and length. This indicates that the acid concentration had a significant effect on the distribution of diameter and length of NCCs. From Table 2, it can be seen that the average dimensions in diameter and length of NCC decreased with increasing both acid concentration and reaction times. In other words, both acid concentration and reaction time had a significant effect on the dimensions of NCC. Furthermore, it can be observed from Table 2 that the average aspect ratios of NCC-6, NCC-8, and NCC-180 were 20.67, 22.29, and 23.81, respectively. This indicates that the aspect ratio of NCC particles was enhanced slightly as both acid concentration and reaction time increased. In addition to hydrolysis conditions, the dimensions and aspect ratio of NCC particles were strongly influenced by sources of cellulose [Habibi et al. 2010]. The 
aspect ratio of obtained NCCs in this study was still lower compared to that of others presented on NCCs from other cellulosic sources, such as sisal (60) [Garcia de Rodriguez et al. 2006], wood (35-58) [Roman and Gray 2005], and tunicate (251-493) [Iwamoto et al. 2009] but higher than cotton (15) [Qi et al. 2009].

Table 2

Average diameter, length, and aspect ratio of NCCs particle hydrolyzed under different conditions measured from TEM images

\begin{tabular}{l|llll|}
\hline Sample & $\begin{array}{l}\text { Average diameter (D) } \\
(\mathrm{nm})\end{array}$ & $\begin{array}{l}\text { Average length (L) } \\
(\mathbf{n m})\end{array}$ & $\begin{array}{l}\text { Average aspect ratio } \\
(\mathrm{L} / \mathrm{D})\end{array}$ \\
\hline NCC-6 & $8.07 \pm 1.73$ & $160.22 \pm 21.59$ & $20.67 \pm 4.87$ \\
\hline NCC-12 & $6.77 \pm 1.30$ & $144.49 \pm 12.64$ & $22.29 \pm 5.53$ \\
\hline NCC-180 & $4.55 \pm 0.89$ & $109.23 \pm 28.97$ & $23.81 \pm 2.06$ \\
\hline 3.5. Thermal Stability & &
\end{tabular}

The thermal stability of raw fiber, CPC, and NCCs produced under different hydrolysis conditions was characterized using thermogravimetric analysis (TGA). The resulting TGA thermograms and derivate thermogravimetric analysis (DTG) curves are shown in Figs. 6 and 7, respectively and the results are presented in Table 3. Figure 6a displays the TGA curves of raw fiber, CPC, and NCCs prepared under different acid concentrations at $45^{\circ} \mathrm{C}$ for $70 \mathrm{~min}$. From Fig. $6 \mathrm{a}$, it can be observed that all samples exhibited a relatively small weight loss in the temperature range of $25-150^{\circ} \mathrm{C}$ which was mainly due to the evaporation and removal of moisture absorbed in the sample. In addition, the weight loss at this temperature might also be attributed to compounds with low molecular weight or volatile material remaining and sticking to the samples during the cellulose purification process [Ishak et al. 2012]. From Fig. 6, it can be seen that the moisture of raw fiber was completely evaporated at a higher temperature (around $150^{\circ} \mathrm{C}$ ) compared to CPC and NCCs. This was associated with the higher moisture content of raw fiber resulting in higher weight loss (15\%) compared to CPC and NCCs [llyas et al. 2018]. Furthermore, the higher moisture content in the raw fiber was due to its high hemicellulose content (13.7\%) where hemicellulose is an amorphous component with very high water absorption property [lshak et al. 2012]. From Fig. 6, it can be also observed that all NCCs and CPC exhibited lower moisture content compared to raw fiber due to the elimination of amorphous regions (hemicellulose, lignin) and dehydration of cellulose fibers during acid hydrolysis.

Figure 7 shows the DTG curves of raw fibers, CPC, and NCCs prepared under different hydrolysis conditions. The onset temperature of major decomposition of raw fiber, CPC, and NCCs is presented in Table 3. The major decomposition of all samples started around $158-254^{\circ} \mathrm{C}$. From Table 3 , it can be seen that the onset temperatures of raw fiber, CPC, and NCCs were 158,242 , and $233-254^{\circ} \mathrm{C}$. The onset temperature of $\mathrm{CPC}$ was higher than the raw fiber due to the lower amorphous region of the CPC resulting in higher thermal stability [Sonia and Dasan 2013]. Furthermore, it can be also seen from Table 3 that both CPC and NCCs had higher thermal stability than the raw fibers. This was again associated with the 
removal of amorphous regions (hemicellulose, lignin) during the chemical purification process and acid hydrolysis as confirmed by the results of FT-IR and XRD previously. The more crystalline component of NCCs compared to both raw fiber and CPC might be believed to be responsible for the higher thermal stability of NCCs [Mohamed et al. 2017]. In addition, the lower decomposition temperature of raw fiber was associated with the low degradation temperature of hemicellulose, lignin, and pectin [Chen et al. 2011; Nasri-Nasrabadi et al. 2014]. Furthermore, the onset temperature of NCC prepared under acid concentrations of 6,8 , and 12 are 254,253 , and $252^{\circ} \mathrm{C}$, respectively. No significant difference in the onset temperatures suggests that the acid concentration did not affect the thermal stability. This is consistent with the results of the crystallinity index as shown in Table 3. The effect of reaction time during hydrolysis on the thermal stability was characterized by the values of the onset temperature of major decomposition. From Table 3, it can be found that the onset temperature of NCCs prepared under different reaction times of 70,125 , and $180 \mathrm{~min}$ is 254,233 , and $235^{\circ} \mathrm{C}$, respectively. It is interesting to note that the onset temperature was decreased drastically with increasing the reaction time. This indicates that the thermal stability of NCC reduced with an increase in reaction time and the best thermal stability was obtained for the hydrolysis time of $70 \mathrm{~min}$. Longer reaction time during hydrolysis led to increase in the number of short cellulose chains due to the highly specific surface are and free end chains of nanocrystalline cellulose and then resulted in the reduced thermal stability [Nasri-Nasrabadi et al. 2012]. These end chains began to degrade at low temperatures [Wang et al. 2007]. Moreover, some damage on the crystal structure due to longer reaction time might be responsible for the reduced thermal stability of NCC [Jiang et al. 2017]. It can also be seen from Table 3 that the final residue left $600{ }^{\circ} \mathrm{C}$ of the NCCs was higher than both the raw fiber and CPC. This was attributed to a higher level of crystallinity of NCCs compared to both raw fiber and CPC. In addition, the higher solid residue observed for NCCs was probably ascribed to the changes of cellulose structure during moisture evaporation at around $100{ }^{\circ} \mathrm{C}$, which reduced the oxygen content in the NCCs, and thus producing carbon structures with higher thermal stability [Kim et al. 2001]. The final residues are affected by several factors including the chemical structure, crystallinity, source of cellulose materials, and hydrolysis conditions for isolation of nanocrystalline cellulose [Oun and Rhim 2016]. From Table 3, it was also found that the maximum degradation temperature $\left(T_{\max }\right)$ of raw fiber, $C P C$, and NCCs were 346,337 , and $328-357^{\circ} \mathrm{C}$, respectively.

The lower $\mathrm{T}_{\max }$ exhibited by CPC compared to the raw fiber was ascribed to the lower amorphous components (hemicellulose, lignin) of CPC due to chemical pretreatments. Further removal of amorphous regions during acid hydrolysis might be believed to responsible for the reduced $T_{\max }$ of NCCs compared to both raw fiber and CPC. From Table 3, it can also be seen that the $T_{\max }$ of NCCs was unchanged with increasing the acid concentration. It was found that the acid concentration did not influence the $T_{\max }$. Furthermore, there was a significant effect from the reaction time where $T_{\max }$ of NCCs was increased with increasing the hydrolysis time.

\section{Conclusion}

Nanocrystalline celluloses (NCCs) were successfully extracted from CPC using hydrochloric acid with varying the acid concentration and reaction time at a constant temperature of $45^{\circ} \mathrm{C}$. The chemical 
structure of cellulose did not change during both chemical treatments and acid hydrolysis processes as confirmed by FT-IR and XRD results. The acid concentration did not affect the crystallinity and thermal stability whereas the reaction time influenced strongly the crystallinity and thermal stability of NCCs. The higher thermal stability was exhibited by NCCs compared to both raw fiber and CPC. The crystallinity index and thermal stability of NCCs were reduced with an increase in the hydrolysis times. The optimal hydrolysis conditions were found to be the reaction time of $70 \mathrm{~min}$ with $6 \mathrm{M}$ hydrochloric acid at $45^{\circ} \mathrm{C}$, resulting in NCC with a rod-like shape with high crystallinity index $(89.61 \%)$, the crystallite size $(5.81 \mathrm{~nm})$, the average diameter $(8.07)$ and average length $(158.51 \mathrm{~nm})$. The obtained NCCs had great potential to be used as reinforcing agents in nanocomposites.

\section{Abbreviations}

$\mathrm{NCC}_{\mathrm{s}}$

nanocrystalline celluloses

NCC

nanocrystalline cellulose

$\mathrm{CPC}$

chemically purified cellulose

NCC-6

nanocrystalline cellulose prepared at $6 \mathrm{M}$ hydrochloric acid at $45^{\circ} \mathrm{C}$ for $70 \mathrm{~min}$ NCC-8

nanocrystalline cellulose prepared at $8 \mathrm{M}$ hydrochloric acid at $45^{\circ} \mathrm{C}$ for $70 \mathrm{~min}$ NCC-12

nanocrystalline cellulose prepared at $12 \mathrm{M}$ hydrochloric acid at $45^{\circ} \mathrm{C}$ for $70 \mathrm{~min}$ $\mathrm{HCl}$

hydrochloric acid

NCC-70

nanocrystalline cellulose prepared at $6 \mathrm{M}$ hydrochloric acid at $45^{\circ} \mathrm{C}$ for $70 \mathrm{~min}$ NCC-125

nanocrystalline cellulose prepared at $6 \mathrm{M}$ hydrochloric acid at $45^{\circ} \mathrm{C}$ for $125 \mathrm{~min}$ NCC-180

nanocrystalline cellulose prepared at $6 \mathrm{M}$ hydrochloric acid at $45^{\circ} \mathrm{C}$ for $180 \mathrm{~min}$

\section{Declarations}

Ethics approval and consent to participate

Not applicable.

\section{Consent for publication}


Not applicable.

\section{Availability of data and materials}

The datasets used and/or analysed during the current study are available from the corresponding author on reasonable request.

\section{Competing interests}

The authors declare that they have no competing interests.

\section{Funding}

This work was financially supported by Universitas Gadjah Mada, Yogyakarta, Indonesia through the research grant Hibah Penelitian Dasar Unggulan Perguruan Tinggi (PDUPT) with the contract no. 110/UN1/DITLIT/DIT-LIT/LIT/2018 and no. 2645/UN1.DITLIT/DIT-LIT/LT/2019.

\section{Authors' contributions}

Kusmono is involved in preparing concept, reviewing the literature, designing experimental work, guiding the analysis, improving and revising the manuscript. Dimas Abdillah Akbar is involved in performing the preparation of the NCCs, doing the characterization of the NCCs and drafting the manuscript. Both the authors are involved in writing the manuscript. All authors read and approved the final manuscript.

\section{Acknowledgments}

Not applicable.

\section{References}

1. Al-Dulaimi AA, Wanrosli WD (2017) Isolation and Characterization of Nanocrystalline Cellulose from Totally Chlorine Free Oil Palm Empty Fruit Bunch Pulp. J Polym Environ 25:192-202

2. Alemdar A, Sain M (2008) Isolation and characterization of nanofibers from agricultural residuesWheat straw and soy hulls. Biores Technol 99(6):1664-1671

3. Bhatnagar A (2005) Processing of cellulose nanofiber-reinforced composites. J Reinf Plast Compos 24(12):1259-1268

4. Bledzki AK, Gassan J (1999) Composites reinforced with cellulose based fibres. Prog Polym Sci 24(2):221-274

5. Brinchi L, Cotana F, Fortunati E, Kenny JM (2013) Production of nanocrystalline cellulose from lignocellulosic biomass: Technology and applications. Carbohyd Polym 94(1):154-169 
6. Brito BSL, Pereira FV, Putaux JL, Jean B (2012) Preparation, morphology and structure of cellulose nanocrystals from bamboo fibers. Cellulose 19(5):1527-1536

7. Chen W, Yu H, Liu Y, Chen P, Zhang M, Hai Y (2011) Individualization of cellulose nanofibers from wood using high-intensity ultrasonication combined with chemical pretreatments. Carbohyd Polym 83(4):1804-1811

8. Cheng M, Qin Z, Chen Y, Hu S, Ren Z, Zhu M (2017) Efficient extraction of cellulose nanocrystals through hydrochloric acid hydrolysis catalyzed by inorganic chlorides under hydrothermal conditions. ACS Sustainable Chemistry Engineering 5(6):4656-4664

9. Cheriana BM, Leão AL, Souza SF, Thomas S, Pothan LA, Kottaisamy M (2010) Isolation of nanocellulose from pineapple leaf fibres by steam explosion. Carbohydrate Polymers 81(3):720-725

10. Corrêa AC, de Teixeira EM, Pessan LA, Mattoso LHC (2010) Cellulose nanofibers from curaua fibers. Cellulose 17(6):1183-1192

11. Ditzel Fl, Prestes E, Carvalho BM, Demiate IM, Pinheiro LA (2017) Nanocrystalline cellulose extracted from pine wood and corncob. Carbohyd Polym 157:1577-1585

12. Dungani R, Owolabi AF, Saurabh CK, Khalil HPSA, Tahir PM, Hazwan CICM, Ajijolakewu KA, Masri MM, Rosamah E, Aditiawati P (2017) Preparation and Fundamental Characterization of Cellulose Nanocrystal from Oil Palm Fronds Biomass. J Polym Environ 25:692-700

13. Fan JS, Li YH (2012) Maximizing the yield of nanocrystalline cellulose from cotton pulp fiber. Carbohyd Polym 88(4):1184-1188

14. Fardioui M, Stambouli A, Gueddira T, Dahrouch A, Qaiss AEK, Bouhfid R (2016) Extraction and Characterization of Nanocrystalline Cellulose from Doum (Chamaerops humilis) Leaves: A Potential Reinforcing Biomaterial. Journal of Polymer the Environment 24:356-362

15. Garcia de Rodriguez NL, Thielemans W, Dufresne A (2006) Sisal cellulose whiskers reinforced polyvinyl acetate nanocomposites. Cellulose 13(3):261-270

16. Habibi Y, Lucia LA, Rojas OJ (2010) Cellulose Nanocrystals: Chemistry, Self-Assembly, and Applications. Chemical reviews 110:3479-3500

17. Ilyas RA, Sapuan SM, Ishak MR (2018) Isolation and characterization of nanocrystalline cellulose from sugar palm fibres (Arenga Pinnata). Carbohyd Polym 181:1038-1051

18. Ishak MR, Sapuan SM, Leman Z, Rahman MZA, Anwar UMK (2012) Characterization of sugar palm (Arenga pinnata) fibres Tensile and thermal properties. J Therm Anal Calorim 109(2):981-989

19. Iwamoto $S$, Kai W, Isogai A, Iwata $T$ (2009) Elastic modulus of single cellulose microfibrils from tunicate measured by atomic force microscopy. Biomacromol 10(9):2571-2576

20. Jasmani L, Adnan S (2017) Preparation and characterization of nanocrystalline cellulose from Acacia mangium and its reinforcement potential. Carbohyd Polym 161:166-171

21. Jiang Y, Zhou J, Zhang Q, Zhao G, Heng L, Chen D, Liu D (2017) Preparation of cellulose nanocrystals from Humulus japonicus stem and the influence of high temperature pretreatment. Carbohyd Polym 164:284-293 
22. Kargarzadeh H, Ahmad I, Abdullah I, Dufresne A, Zainudin SY, Sheltami RM (2012) Effects of hydrolysis conditions on the morphology, crystallinity, and thermal stability of cellulose nanocrystals extracted from kenaf bast fibers. Cellulose 19:855-866

23. Khalil HPSA, Bhat AH, Yusra AFI (2012) Green composites from sustainable cellulose nanofibrils: A review. Carbohyd Polym 87(2):963-979

24. Kian L, Jawaid M, Arif H, Karim Z (2018) Isolation and characterization of nanocrystalline cellulose from roselle-derived microcrystalline cellulose. Int J Biol Macromol 114:54-63

25. Kim DY, Nishiyama Y, Wada M, Kuga S (2001) High-yield Carbonization of Cellulose by Sulfuric Acid Impregnation. Cellulose 8(1):29-33

26. Kumar A, Negi YS, Choudhary V, Bhardwaj NK (2014) Characterization of Cellulose Nanocrystals Produced by Acid-Hydrolysis from Sugarcane Bagasse as Agro-Waste. Journal of Materials Physics Chemistry 2(1):1-8

27. Lei W, Fang C, Zhou X, Yin Q, Pan S, Yang R, Ouyang Y (2018) Cellulose nanocrystals obtained from office waste paper and their potential application in PET packing materials. Carbohyd Polym 181:376-385

28. Lei W, Fang C, Zhou X, Yin Q, Pan S, Yang R, Ouyang Y (2018) Cellulose nanocrystals obtained from office waste paper and their potential application in PET packing materials. Carbohyd Polym 181:376-385

29. Lima MMS, Borsali R (2004) Rod like cellulose microcrystals: structure, properties and applications. Macromolecular Rapid Communication 25:771-787

30. Listyanda RF, Kusmono, Wildan MW, IIman MN (2020) Extraction and characterization of nanocrystalline cellulose (NCC) from ramie fiber by sulphuric acid hydrolysis. AIP Conference Proceedings 2217:030069

31. Liu Y, Wang H, Yu G, Yu Q, Li B, Mu X (2014) A novel approach for the preparation of nanocrystalline cellulose by using phosphotungstic acid. Carbohyd Polym 110:415-422

32. Liu Z, Li X, Xie W, Deng H (2017) Extraction, isolation and characterization of nanocrystalline cellulose from industrial kelp (Laminaria japonica) waste. Carbohyd Polym 173:353-359

33. Lu P, Hsieh YL (2012) Preparation and characterization of cellulose nanocrystals from rice straw. Carbohyd Polym 87(1):564-573

34. Mendes CAC, Ferreira NMS, Furtado RS, Sousa AMF (2015) Isolation and characterization of nanocrystalline cellulose from corn husk. Mater Lett 148:26-29

35. Mishra S, Kharkar PS, Pethe AM (2019) Biomass and waste materials as potential sources of nanocrystalline cellulose: Comparative review of preparation methods (2016 - Till date). Carbohyd Polym 207:418-427

36. Mohamed MA, Salleh WNW, Jaafar J, Ismail AF, Mutalib MA, Mohamad AB, Mohd Hir ZA (2017) Physicochemical characterization of cellulose nanocrystal and nanoporous self-assembled CNC membrane derived from Ceiba pentandra. Carbohyd Polym 157:1892-1902 
37. Morais JPS, Rosa MDF, de Souza Filho, desá M, Nascimento LD, do Nascimento DM, Cassales AR (2013) Extraction and characterization of nanocellulose structures from raw cotton linter. Carbohydrate Polymers 91(1):229-235

38. Moriana R, Filaplana F, Ek M (2016) Cellulose Nanocrystals from Forest Residues as Reinforcing Agents for Composites: A Study from Macro-to Nano-Dimensions. Carbohyd Polym 139:139-149

39. Munawar SS, Umemura K, Tanaka F (2008) Effects of alkali, mild steam, and chitosan treatments on the properties of pineapple, ramie, and sansevieria fiber bundles. Journal of Wood Science 54(1):2835

40. Naduparambath S, Jinitha TV, Shaniba V, Sreejith MP, Balan AK, Purushothaman E (2018) Isolation and characterisation of cellulose nanocrystals from sago seed shells. Carbohyd Polym 180:13-20

41. Nasri-Nasrabadi B, Behzad TT, Bagheri R (2014) Extraction and characterization of rice straw cellulose nanofibers by an optimized chemomechanical method. J Appl Polym Sci 131:40063

42. Neto WPF, Silvério HA, Dantas DN, Pasquini D (2013) Extraction and characterization of cellulose nanocrystals from agro-industrial residue-Soy hulls. Ind Crops Prod 42:480-488

43. Ngwabebhoh FA, Erdem A, Yildiz U (2018) A design optimization study on synthesized nanocrystalline cellulose, evaluation and surface modification as a potential biomaterial for prospective biomedical applications. Int J Biol Macromol 114:536-546

44. Normand ML, Moriana R, Ek M (2014) Isolation and characterization of cellulose nanocrystals from sprucebark in a biorefinery perspective. Carbohyd Polym 111:979-987

45. Oun AA, Rhim JW (2015) Effect of post-treatments and concentration of cotton linter cellulose nanocrystals on the properties of agar-based nanocomposite films. Carbohyd Polym 134:20-29

46. Oun AA, Rhim JW (2016) Isolation of cellulose nanocrystals from grain straws and their use for the preparation of carboxymethyl cellulose-based nanocomposite films. Carbohyd Polym 150:187-200

47. Owolabi AWT, Ghazali A, Wanrosli WD, Annas FMA (2016) Effect of alkaline peroxide pre-treatment on microfibrillated cellulose from oil palm fronds rachis amenable for puplp and paper and biocomposite production. BioResources 11(2):3013-3026

48. Peng BL, Dhar N, Liu HL, Tam KC (2011) Chemistry and applications of nanocrystalline cellulose and its derivatives: A nanotechnology perspective. Can J Chem Eng 89(5):1191-1206

49. Pirani S, Hashaikeh R (2013) Nanocrystalline cellulose extraction process and utilization of the byproduct for biofuels production. Carbohyd Polym 93(1):357-363

50. Qi H, Cai J, Zhang L, Kuga S (2009) Properties of films composed of cellulose nanowhiskers and a cellulose matrix regenerated from alkali/urea solution. Biomacromol 10(6):1597-1602

51. Roman M, Gray DG (2005) Parabolic focal conics in self-assembled solid films of cellulose nanocrystals. Langmuir 21(12):5555-5561

52. Roman M, Winter WT (2004) Effect of sulfate groups from sulfuric acid hydrolysis on the thermal degradation behavior of bacterial cellulose. Biomacromol 5:1671-1677 
53. Rosa MF, Medeiros ES, Malmonge JA, Gregorski KS, Wood DF, Mattoso LHC, Imam SH (2010) Cellulose nanowhiskers from coconut husk fibers: Effect of preparation conditions on their thermal and morphological behavior. Carbohyd Polym 81(1):83-92

54. Rosli NA, Ahmad I, Abdullah I (2013) Isolation and characterization of cellulose nanocrystals from Agave angustifolia fibre. BioResources 8(2):1893-1908

55. Samir MASA, Alloin F, Paillet M, Dufresne A (2004) Tangling Effect in Fibrillated Cellulose Reinforced Nanocomposites. Macromolecules 37:4313-4316

56. Segal L, Creely JJ, Martin AE, Conrad CM (1959) An empirical method for estimating the degree of crystallinity of native cellulose using the X-Ray Diffractometer. Text Res J 29:786-794

57. Sheltami RM, Abdullah I, Ahmad I, Dufresne A, Kargarzadeh H (2012) Extraction of cellulose nanocrystals from mengkuang leaves (Pandanus tectorius). Carbohyd Polym 88(2):772-779

58. Sonia A, Dasan KP (2013) Chemical, morphology and thermal evaluation of cellulose microfibers obtained from Hibiscus sabdariffa. Carbohyd Polym 92(1):668-674

59. Sun Q, Zhao X, Wang D, Dong J, She D, Peng P (2018) Preparation and characterization of nanocrystalline cellulose/Eucommia ulmoides gum nanocomposite film. Carbohyd Polym 181:825832

60. Tang YJ, Yang SJ, Zhang N, Zhang JH (2014) Preparation and characterization of nanocrystalline cellulose via low-intensity ultrasonic-assisted sulfuric acid hydrolysis. Cellulose 21:335-346

61. Wang N, Ding E, Cheng R (2007) Thermal degradation behavior of spherical cellulose nanocrystals with sulfate groups. Polymer 48:3486-3493

62. Wang Z, Yao Z, Zhou J, He M, Jiang Q, Li S, Ma Y (2019) Isolation and characterization of cellulose nanocrystals from pueraria root residue. Int J Biol Macromol 129:1081-1089

63. Yu H, Qin Z, Liang B, Liu N, Zhou Z, Chen L (2013) Facile extraction of thermally stable cellulose nanocrystals with a high yield of $93 \%$ through hydrochloric acidhydrolysis under hydrothermal conditions. Journal of Materials Chemistry A 12:3938-3944

64. Zainuddin N, Ahmad I, Kargarzadeh H, Ramli S (2017) Hydrophobic kenaf nanocrystalline cellulose for the binding of curcumin. Carbohyd Polym 163:261-269

\section{Figures}




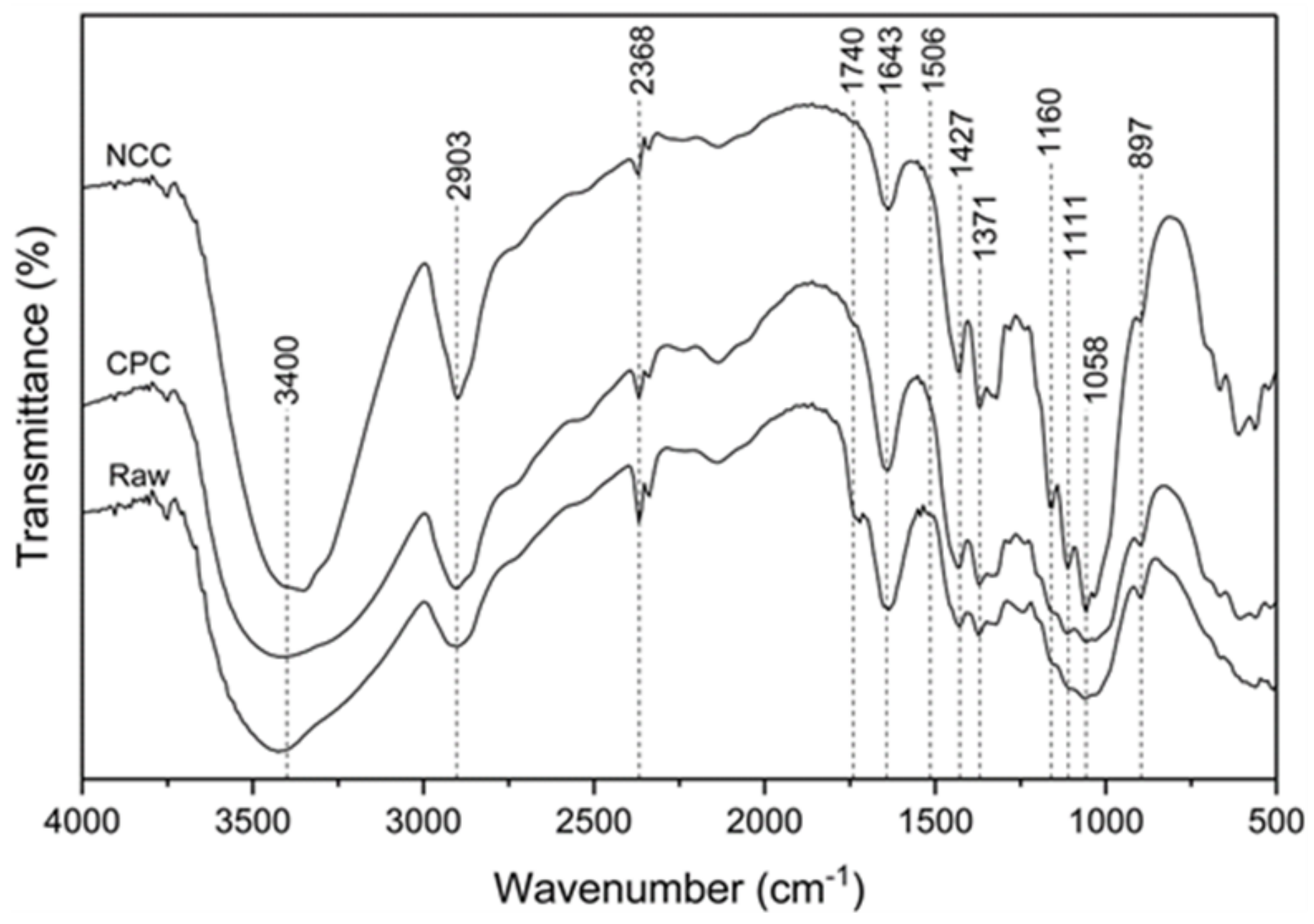

Figure 1

FT-IR spectra of raw fibers, CPC, and NCC
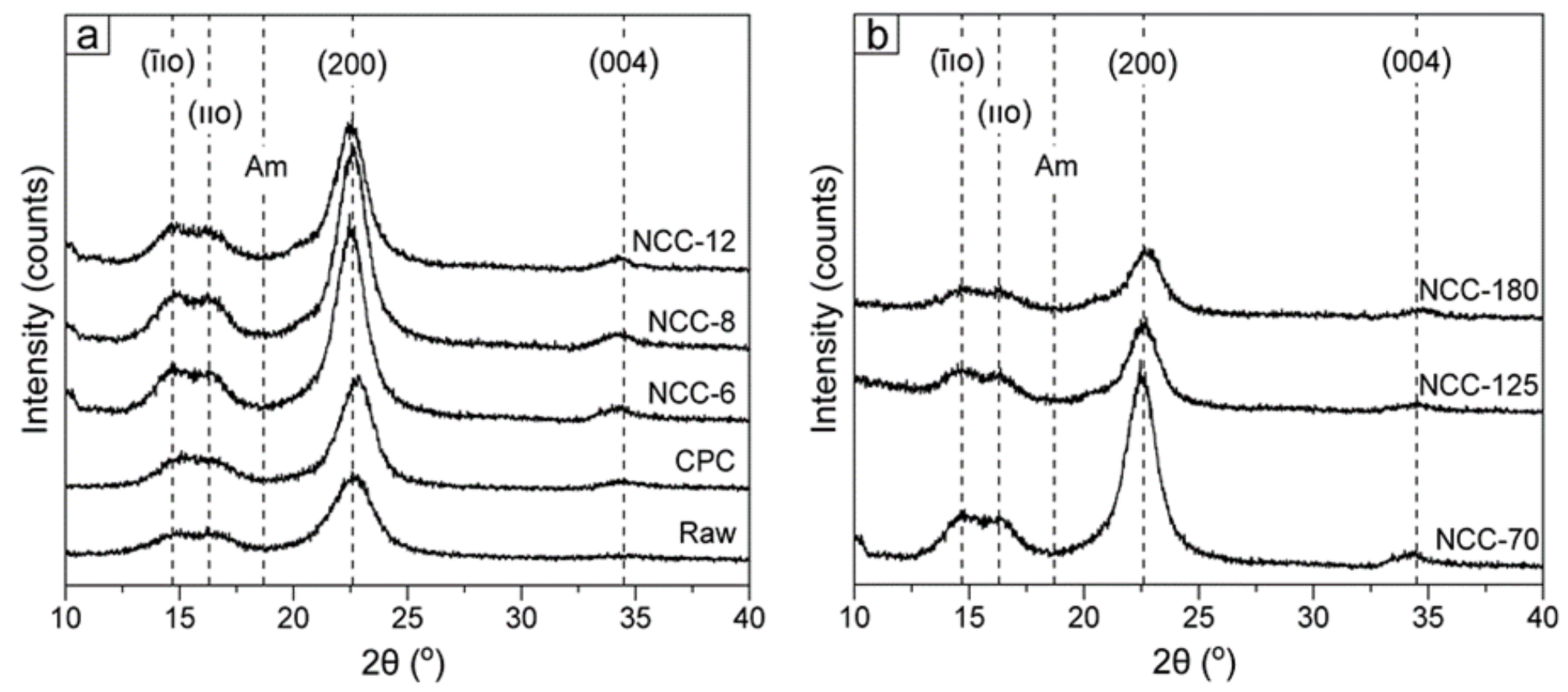
Figure 2

XRD patterns of raw fiber, CPC, and NCCs: a. Under different acid concentrations b. Under different reaction times
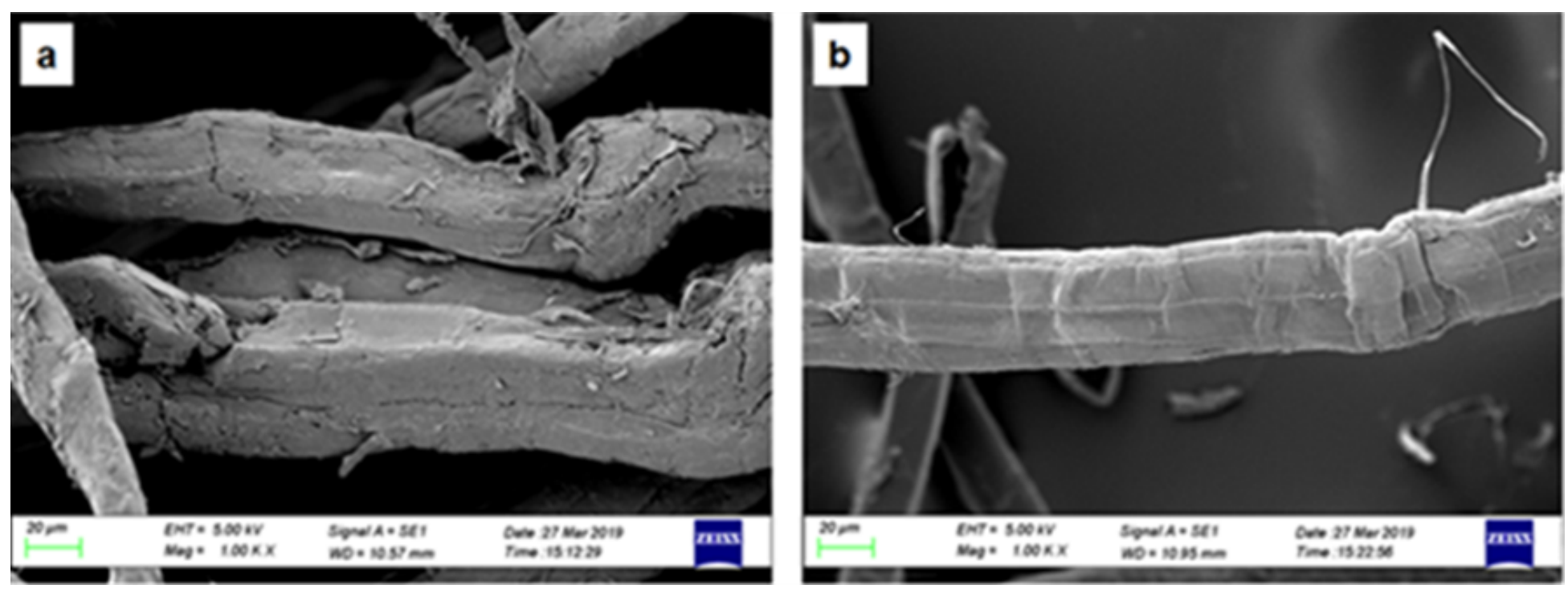

Figure 3

SEM micrographs of a. Raw fibers b. CPC
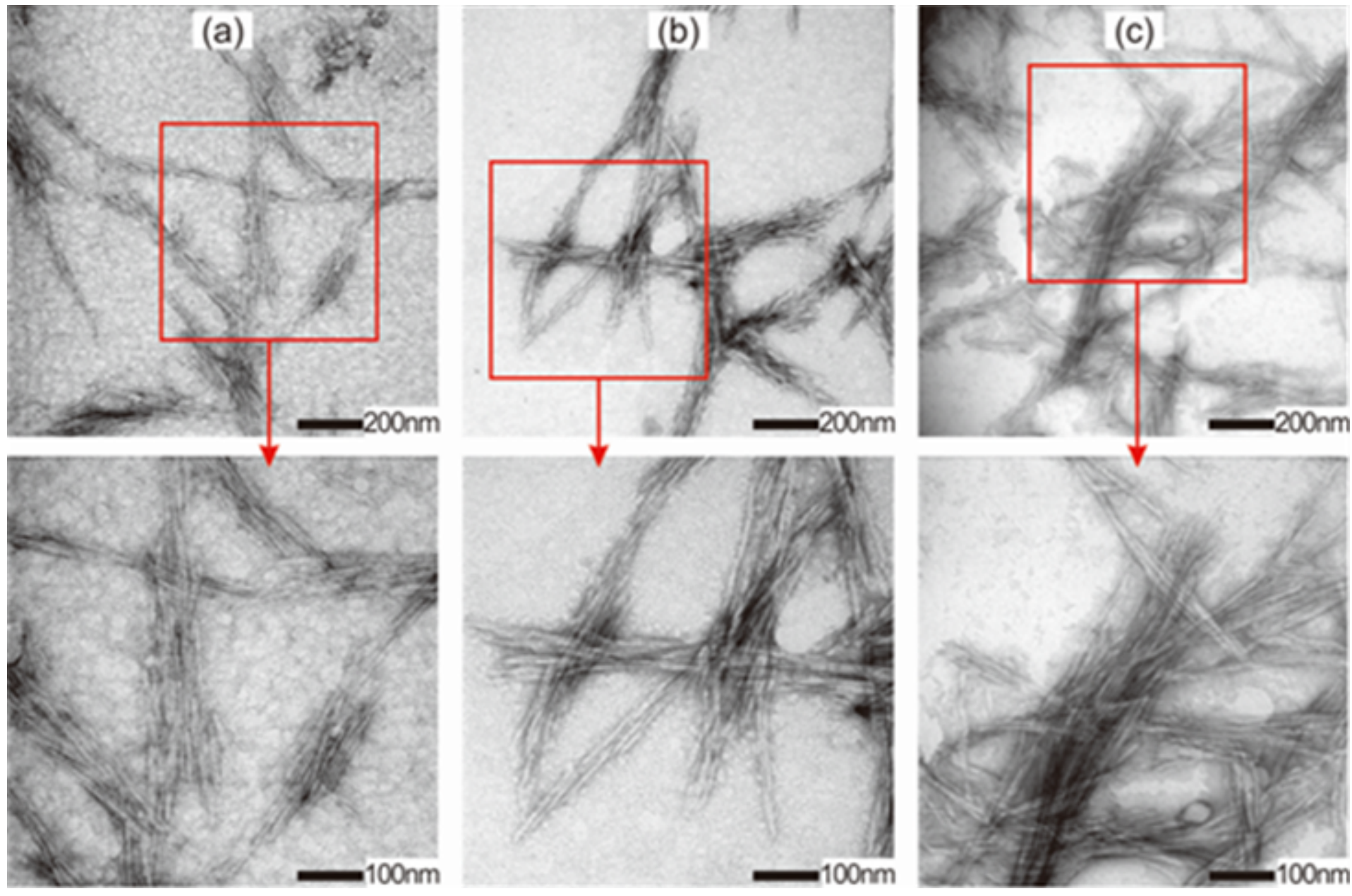
Figure 4

TEM images of NCCs under different hydrolysis conditions: a. NCC-6 b. NCC-12 c. NCC-180
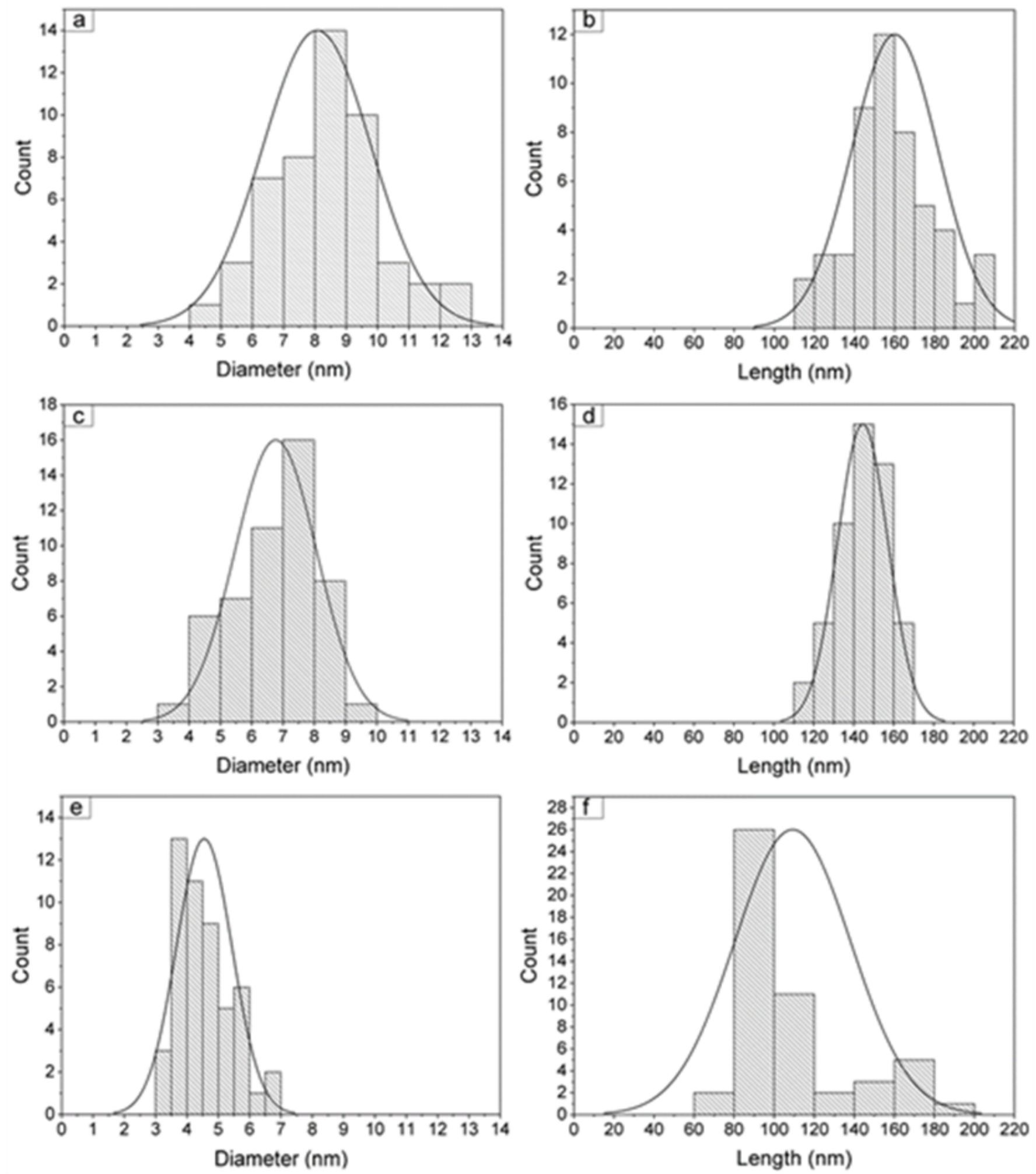

Figure 5

Diameter and length distribution of NCCs under different hydrolysis conditions: $(a, b)$ NCC-6 (c,d) NCC-12; $(e, f)$ NCC-180 

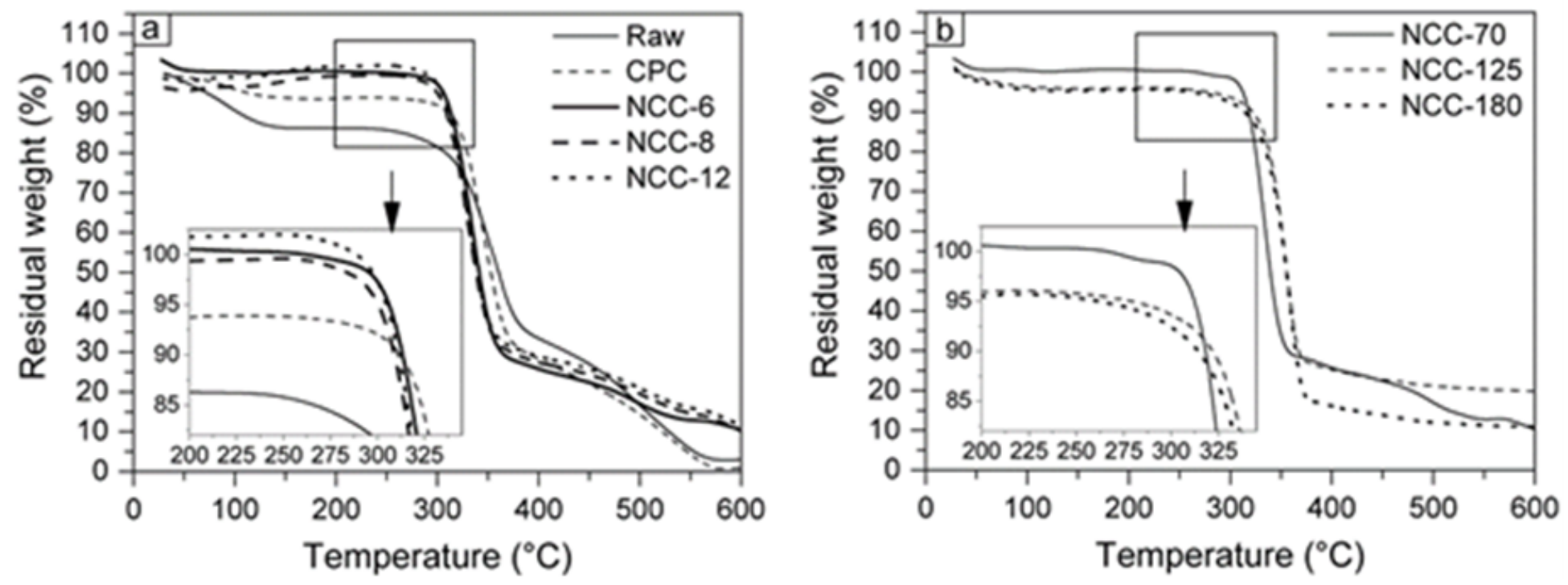

Figure 6

TGA curves of: a. Raw, CPC, and NCCs prepared under different acid concentrations b. NCCs prepared under different reaction times
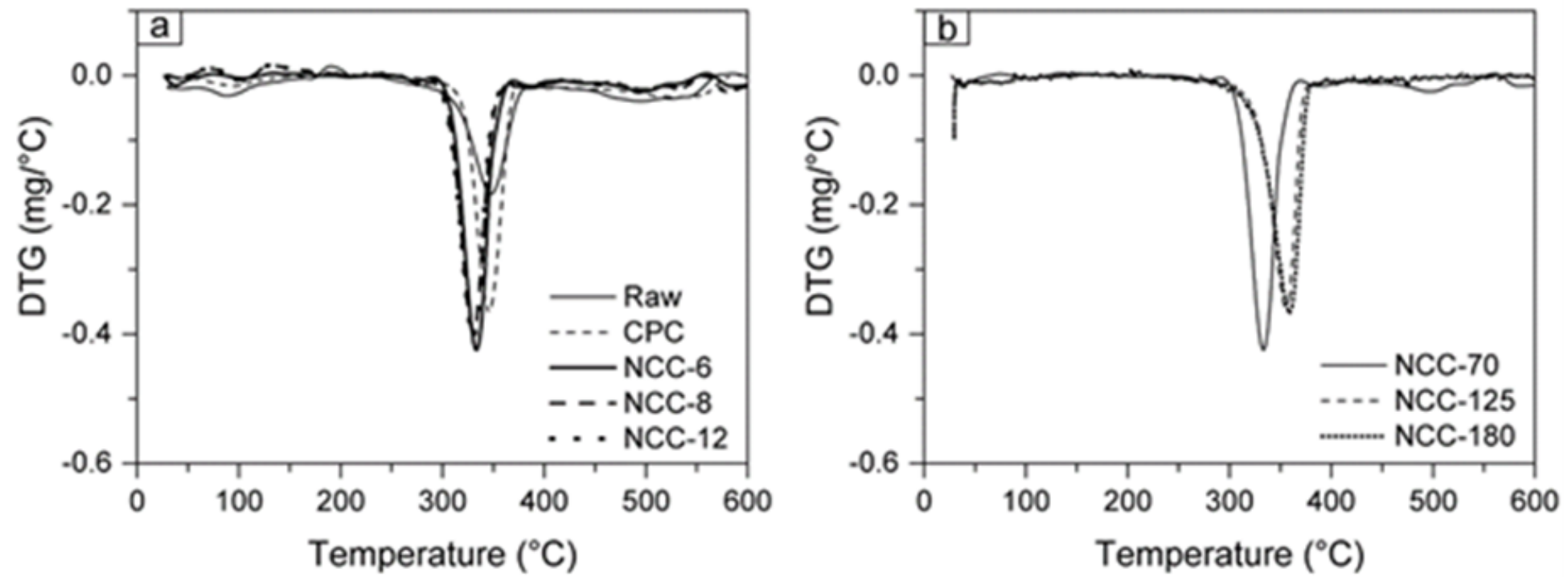

Figure 7

DTG curves of: a. Raw, CPC and NCCs prepared under different acid concentration b. NCCs prepared under different reaction time

\section{Supplementary Files}

This is a list of supplementary files associated with this preprint. Click to download.

- Graphicalabstract.jpg 